\title{
Study of distance range visual measurement in the new Depth From Defocus method
}

\author{
K. Murawski, ${ }^{* 1}$ A. Arciuch, ${ }^{1}$ T. Pustelny ${ }^{2}$ \\ ${ }^{1}$ Institute of Teleinformatics and Automatics, Military University of Technology, Kaliskiego 2, 00-908 Warsaw, \\ ${ }^{2}$ Department of Optoelectronics, Silesian University of Technology, Krzywoustego 2, 44-100 Gliwice
}

Received May 30, 2016; accepted June 23, 2016; published June 30, 2016

\begin{abstract}
The article presents the results achieved while researching a distance measuring method belonging to Depth From Defocus techniques. The method has been developed to determine the shape of a flaccid diaphragm used in the Ventricular Assist Device (VAD). The shape is determined on the basis of distance measured between the CCD sensor plate of a camera and the markers located on the flaccid diaphragm. The goal of this paper is to present the influence of marker size on distance range measured between the camera and the diaphragm used in a pneumatic prosthetic heart.
\end{abstract}

The article presents the impact of marker size on the result of a distance measurement method $[1,2]$. The method was developed for a video sensor to determine the instantaneous stroke volume of blood $S V$ from a pneumatic pump heart assist device $(V A D)$, Fig. 1a. When measuring $S V$, the flaccid diaphragm is equipped with markers which position and orientation in 3D space is subject to change in relation to the camera position. What is important in the method is that when measuring the position of the camera and all settings: the lens and the camera (focus, aperture, focal length) are invariable. Method [1,2] also defines in 3D space the position of all the markers on the basis of analysis of only one image frame. In this respect, it has no equivalent in the literature. The vision systems mentioned in the literature typically create a stereoscopic system [3]. The distance in such a system is calculated knowing the optical parameters of the cameras and their mutual position. Measuring systems equipped with one camera are also used [4]. The distance to the object in these systems is determined using, for example, a perspective inverse transform [5]. A single camera configuration with an autofocus function is also possible. The distance to the object is then determined with a lens equation [6]. Other methods used to measure the distance are photogrammetry [7-8] and a fringe projection technique [9-11]. Their use, however, depends on: the scope of measured distances, required measurement speed, required image sensor resolution, light source type as well as target size and sensor weight.

When determining the shape of a flaccid diaphragm, real time operation is particularly important (the shape of the diaphragm may change with a frequency of up to $3 \mathrm{~Hz}$ ) as

\footnotetext{
*E-mail: Krzysztof.Murawski@wat.edu.pl
}

well as simultaneous multipoint measurement of the position in $3 \mathrm{D}$ space in respect to all of the markers, Fig. 1b.

The development of a soft-sensor for measuring the $S V$ of a pulsating heart assist pump results from the needs and problems mentioned in [12]. In [12-13] it was demonstrated that $S V$ can be determined by using the acoustic Helmholtz resonator theory. This method, however, has substantial limitations. For this reason the search for techniques of determining $S V$ is still valid. The knowledge of changes dynamics in $S V$ will enhance the safety of $V A D$ usage, which currently depends on visual inspection: "One of the main advantages of the extracorporeal, polyurethane blood pump is its transparency that allows running continuous visual inspection of the pump state and the quality of its work." [12].As an alternative to the current state, it is proposed to use augmented reality. In [14] a technique was presented according to which the diaphragm image is determined from $\mathrm{f} \geq 5 \mathrm{~Hz}$ : coordinates of diaphragm point nodes; visualization of diaphragm shape in 3D space. Exemplary results of the reconstruction of the diaphragm shape are shown in Fig. 2. The reconstruction accuracy of the diaphragm using technique [14] depends on the number of markers and the precision of distance determination. For this reason, it is advisable to examine the influence of the marker on the accuracy of distance measurement with this particular technique $[1,2]$.

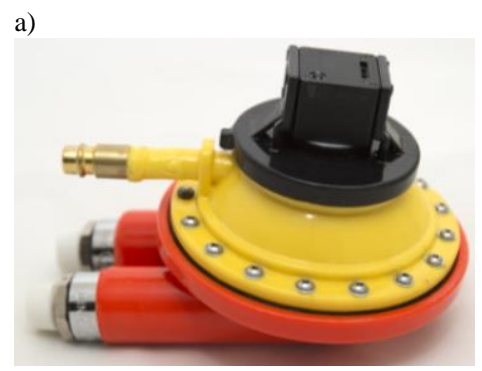

b)

Fig. 1. Model VAD (a), membrane and markers view (b). 


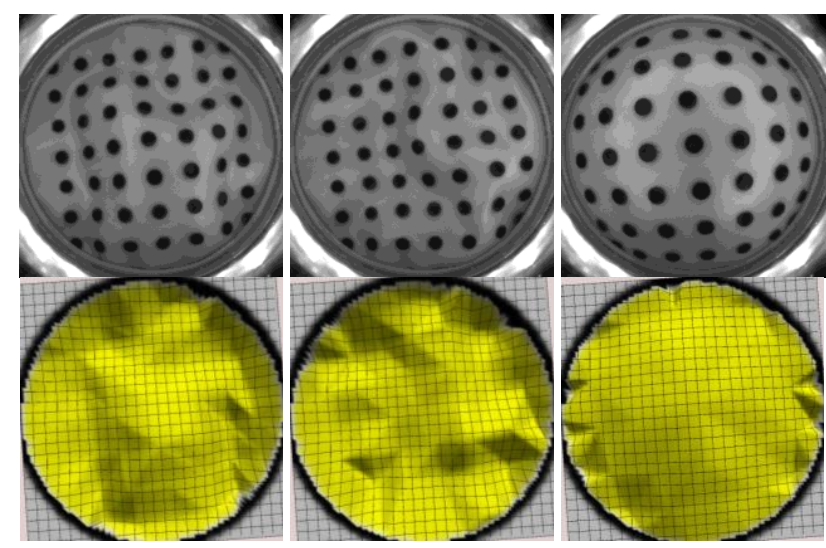

Fig. 2. Reconstruction of the flaccid diaphragm view based on one image using the technique given in [14].

The influence of marker size on distance accuracy and range was studied using an Optitrack v100 slim camera. The camera has a lens with a focal length of $16 \mathrm{~mm}$, visible light filter, infrared radiator and radiator driver. The operation of the controller and IR radiator is discussed in [15-16]. The experiment was performed in the configuration shown in [1-2]. The image sharpness was set at a distance of $d_{0}=0.14 \mathrm{~m}$, measuring from the plane of the camera image sensor. This position is taken as position zero. With respect to it all measurements were made within the range $\left[d_{0}-0.035 \mathrm{~m}, d_{0}+0.035 \mathrm{~m}\right]$, which includes possible displacements of the flaccid diaphragm face occurring in the heart prosthesis. The distance measurement was performed in accordance to the method given in [1-2]. Black circles were used as markers with diameters ranging from $0.003 \mathrm{~m}$ to $0.009 \mathrm{~m}$. The position of the plane with the marker, relative to $d_{0}$, was determined by a stepper motor in increments of $\pm 0.001 \mathrm{~m}$.

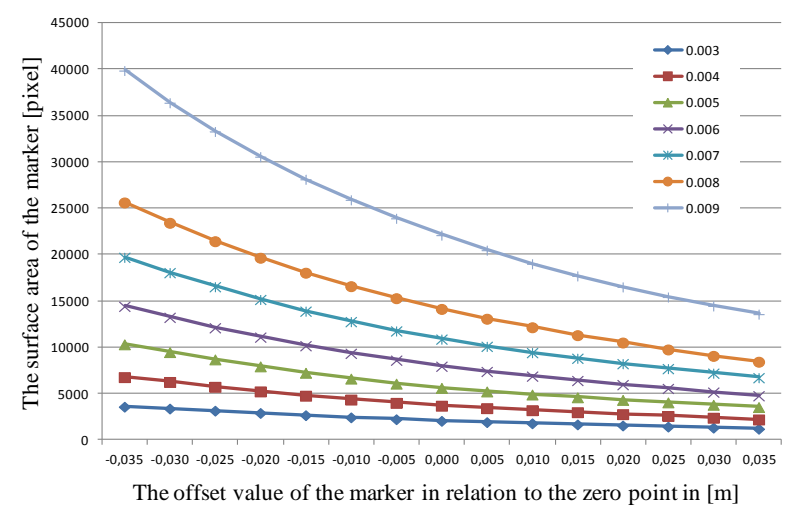

Fig. 3. Measurement results of the variability of surface areas of the markers before standardization

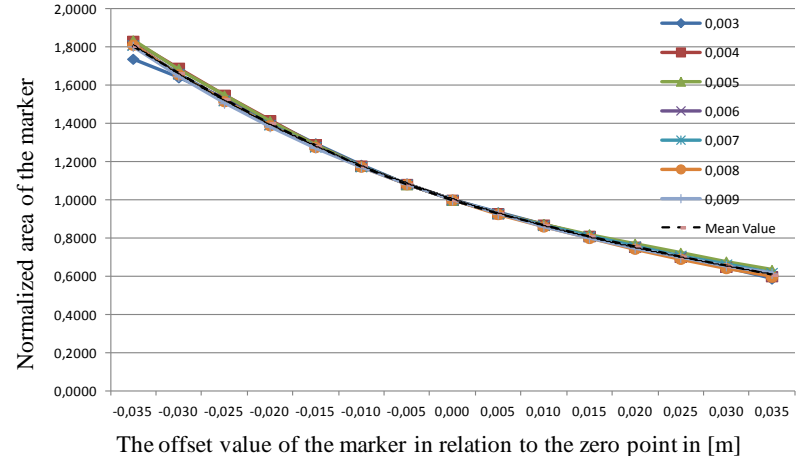

Fig. 4. Measurement results of the variability of surface areas of the markers after standardization.

The study involved determining the influence of marker size on distance measurement accuracy. For this purpose, the dependence was determined of an initial marker size on the observed range of changes in its surface within the accepted range. Measurements were performed for the markers having a diameter from $0.003 \mathrm{~m}$ to $0.009 \mathrm{~m}$ in $0.001 \mathrm{~m}$ increments. Markers are displaced in the range [ $\left.d_{0}-0.035 \mathrm{~m}, d_{0}+0.035 \mathrm{~m}\right]$ in $0.001 \mathrm{~m}$ increments. For each position of the marker, 100 measurements were made of its surface area in accordance with the technique used in [1-2]. The result was determined by calculating the arithmetic mean of the series of measurements. The values of the marker surface areas (obtained before normalization) for position $d_{0}$ were respectively: 2.070, 3.707, 5.632, 7.962, 10.894, 14.156, and 22.122. The resulting area variability of markers in the adopted measuring range is shown in Fig. 3. The results of the experiment obtained after normalization were shown in Fig. 4. Figure 3 shows that the smallest variability of 2.383px (reference value) was obtained for the marker with a diameter of $0.003 \mathrm{~m}$. The marker of the given diameter, although the most promising because of the possibility of high density markers on the diaphragm surface due to relatively small changes in the surface area, does not provide an adequate basis for conducting precise distance measurement. The change in the marker surface with a diameter of $0.003 \mathrm{~m}$ as a distance function is determined with function $f(x)=6.8 x^{2}-279.4 x+3906.4$. A similar result was obtained for markers with a diameter of $0.004 \mathrm{~m}$ and $0.005 \mathrm{~m}$. Their surface area variability amounted to $4.571 \mathrm{px}$ and $6.773 \mathrm{px}$ and the nature of changes is specified with functions $f(x)=$ $16.4 x^{2}-579.8 x+7326.2$ and $f(x)=26.8 x^{2}-898.1 x+11148$. Qualitatively better results were obtained for markers with diameters of $0.006 \mathrm{~m}, 0.007 \mathrm{~m}, 0.008 \mathrm{~m}$ and $0009 \mathrm{~m}$. Changes in the surface areas are defined by functions: $f(x)=-1.5 x^{3}+70.4 x^{2}-1445.7 x+15841$ for $0.006 \mathrm{~m}$; $f(x)=-1.9 x^{3}+94.0 x^{2}-1966.5 x+21631$ for $0.007 \mathrm{~m}$; $f(x)=-2.6 x^{3}+120.7 x^{2}-2541 x+28071$ for $0.008 \mathrm{~m}$ and $f(x)=-3.6 x^{3}+179.4 x^{2}-3887 x+43535$ for $0.009 m$. The 
obtained results, Fig. 3, show that the best marker for measuring the distance was the marker with a diameter of $0.009 \mathrm{~m}$. Given the significant size of the marker, it must be assumed that it is not suitable for determining the shape of the flaccid diaphragm of $V A D$. This marker does not allow creating sufficiently high density markers on the surface of the diaphragm. It does not guarantee proper shape reconstruction.

Another analysis of the results obtained showed that the normalization of the results relative to the surface area of the marker set in point $d_{0}$ introduces a significant and beneficial change in the acquired ranges of variability of the surface areas of the markers, Fig. 4 - "Mean Value" graph. Implementing standardization causes the diameter of a marker positioned on the diaphragm surface, over a distance range, not to have a substantial impact on measurement accuracy and range. The shape of the "Mean Value" graph, Fig. 4, is described by the polynomial $f(x)=-0.025 x^{3}+0.124 x^{2}-0.238 x+0.140$, where $x$ indicates the normalized surface area of the marker. In conclusion, the marker with a diameter of $0.003 \mathrm{~m}$ can be used after calibrating the video sensor to determine the shape of a flaccid $V A D$ diaphragm.

The paper discusses the test results of the author's Depth From Defocus technique [1-2], which was developed to determine the shape of a flaccid diaphragm in real time, Fig. 2, and the stroke volume of a pneumatic heart assist pump, Fig. 1a. Therefore it was especially important to investigate the influence of marker size on the result of distance measurement in the range of movements, to which the face of the diaphragm is subject in the heart prosthesis model. The tests were carried out in the range from $\left[d_{0}-0.035 \mathrm{~m}, d_{0}+0.035 \mathrm{~m}\right]$, where $d_{0}$ indicates the distance of the marker from the plane of the image sensor, for which the focus was set. The study was performed for markers with diameters from $0.003 \mathrm{~m}$ to $0.009 \mathrm{~m}$ in $0.001 \mathrm{~m}$ increments.

The obtained results lead to the following recommendations:

1. Using the technique of measuring the distances shown in $[1,2]$, when normalization of obtained results is not carried out, it is recommended to use markers with a diameter of no less than $0.006 \mathrm{~m}$.

2. The normalization of a marker surface area enables the use of markers with a diameter less than $0.006 \mathrm{~m}$.

3. It is recommended to use the normalization of a marker surface area in order to make distance measurement results independent from the marker diameter and determining the distance from the following equation: $f(x)=-0.025 x^{3}+0.124 x^{2}-0.238 x+$ +0.140 , where $x$ is the normalized surface area of the marker.

\section{References}

[1] K. Murawski, Acta Phys. Pol. A 127(6), 1591 (2015).

[2] K. Murawski, Method of measuring the distance using the cameras, Patent Application No. P.408076 (2014) - in Polish.

[3] H. Wang, J. Hu, Opt. Engin. 51(6), 1 (2012).

[4] SY. Chen, YF. Li, IEEE Trans. Industrial Infor. 9(3), 1680 (2013).

[5] F. Bonin-Font, A. Burguera, A. Ortiz, G. Oliver, Robotica 31, 225 (2013).

[6] A. Bourdonnaye, R. Doskočil, V. Křivánek, A. Štefek, Adv. Military Techn. 7(2), 49 (2012).

[7] http://tdserver1.fnal.gov/darve/mu_cool/pressuretest/Basics_of_Ph otogrammetry.pdf, (2015).

[8] K. Yue, Z. Li, M. Zhang, S. Chen, Opt. Expr. 18(26), 26866 (2010).

[9] Y. Morimoto, A. Masaya, M. Fujigaki, D. Asai, Appl. Measur. Systems 7, 137 (2012).

[10] R.B. Rusu, A. Aldoma, S. Gedikli, M. Dixon, Tutorial at IEEE/ RSJ International Conference on Intelligent Robots and Systems (IROS 2011).

[11] A. Saxena, H. Koppula, R. Newcombe, X. Ren, Workshop in conjunction with Robotics: Science and Systems (RSS 2013).

[12] J. Sarna, R. Kustosz, E. Woźniewska, M. Gonsior, A. Jarosz, K. Szymańska, D. Hansel, E. Krzak, Program "Polskie Sztuczne Serce" Sojusz Medycyny, Nauki i Techniki, Epigraf s.c., Zabrze, 1-240 (2013).

[13] P. Gibinski, G. Konieczny, E. Maciak, Z. Opilski, T. Pustelny, Acoustic device for measuring instantaneous blood volume in cardiac support chamber i.e. pneumatic heart assist driving chamber, has sensor supporting heart in openings, and audio amplifier connected with volume unit of blood-cell support, Patent No. PL394074-A1.

[14] K. Murawski, T. Pustelny, M. Murawska, System and method of determining the shape of diaphragm of pneumatic extracorporeal heart assist pump, Patent Application No. P.414104, (2015) in Polish.

[15] K. Różanowski, K. Murawski, Acta Phys. Pol. A 122(5), 874 (2012).

[16] K. Murawski, D. Białas, M. Rękas, Acta Phys. Pol. A 127(6), 1732 (2015). 